\title{
Surgical Treatment of Habitual Patella Dislocation with Genu Valgum
}

\author{
Ji Hoon Kwak, MD, Jae Ang Sim, MD, Nam Ki Kim, MD and Beom Koo Lee, MD \\ Department of Orthopedics, Gil Medical Center, Gachon University of Medicine and Science, Incheon, Korea
}

\begin{abstract}
Habitual dislocation of patella is a rare disorder. Sometimes it is associated with angular deformity such as genu valgum. We experienced habitual patella dislocation associated with genu valgum that was treated with corrective osteotomy of distal femur and soft tissue realignment procedure including lateral release and medial reefing.
\end{abstract}

Key words: Habitual patella dislocation, Genu valgum, Distal femoral osteotomy.

Habitual patellar dislocation is a rare condition where the patella dislocates during flexion and relocates during extension unlike chronic patellar dislocation that occurs during both flexion and extension of the knee, and it usually presents without pain or swelling.

A variety of surgical techniques have been introduced for the treatment of habitual dislocation of the patella with genu valgum. Among them, osteotomy combined with proximal soft tissue realignment procedures including lateral release and medial reefing has been commonly performed. It has been known that significant treatment results can be obtained with combined procedures, not with a single procedure.

We here report a case of habitual dislocation of the patella with genu valgum treated with a corrective osteotomy of the distal femur combined with lateral release and medial reefing.

Received October 22, 2009; Revised December 15, 2009;

Accepted June 13, 2011.

Correspondence to: Beom Koo Lee, MD.

Department of Orthopedics, Gil Medical Center, Gachon University of Medicine and Science, 1198 Guwol-dong, Namdong-gu, Incheon 405760 , Korea.

Tel: +82-32-460-3384, Fax: +82-32-468-5437

Email: jihoon1976@gmail.com

This is an Open Access article distributed under the terms of the Creative Commons Attribution Non-Commercial License (http://creativecommons.org/licenses/by-nc/3.0/) which permits unrestricted non-commercial use, distribution, and reproduction in any medium, provided the original work is properly cited.

Copyright @ 2011. THE KOREAN KNEE SOCIETY www.jksrr.org
The clinical and radiological outcomes of the treatment were satisfactory.

\section{Case Report}

A 23-year-old man visited our institution due to lateral dislocation of the left knee in flexion and instability that had persisted for 7 years (Fig. 1). The patient had a history of femoral growth plate fracture treated conservatively at 13 years of age. In the physical examination, the apprehension test was positive. In the radiographic examination, the congruence angle, sulcus angle, Q-angle, and Insall-salvati Ratio was $44.17^{\circ}, 152^{\circ}, 18^{\circ}$, and 1.15 , respectively. The tibiofemoral angle measured from the weightbearing radiograph was $16^{\circ}$, indicating genu valgum deformity, and hypoplasia of the lateral femoral condyle of the left knee was observed. A skyline view of the knee showed lateral dislocation of the patella. Arthroscopic examination revealed the hypoplasia of the lateral femoral condyle, Outerbridge grade II cartilage damage on the lateral articular surface, and contracture of the lateral retinaculum and vastus lateralis. Patellar dislocation during flexion was confirmed with arthroscopy. A lateral retinacular release and an open-wedge distal fermoral varus osteotomy were followed by additional procedures including lateral release and medial reefing because dislocation was still present after the osteotomy. About $2 \mathrm{~cm}$ of the medial retinaculum was reefed because it was excessively elongated. When lateral dislocation of the patella in flexion was no longer observed, a bone graft was inserted into the osteotomy site. Gradual flexion exercises were started postoperatively. Complete flexion was obtained at the 

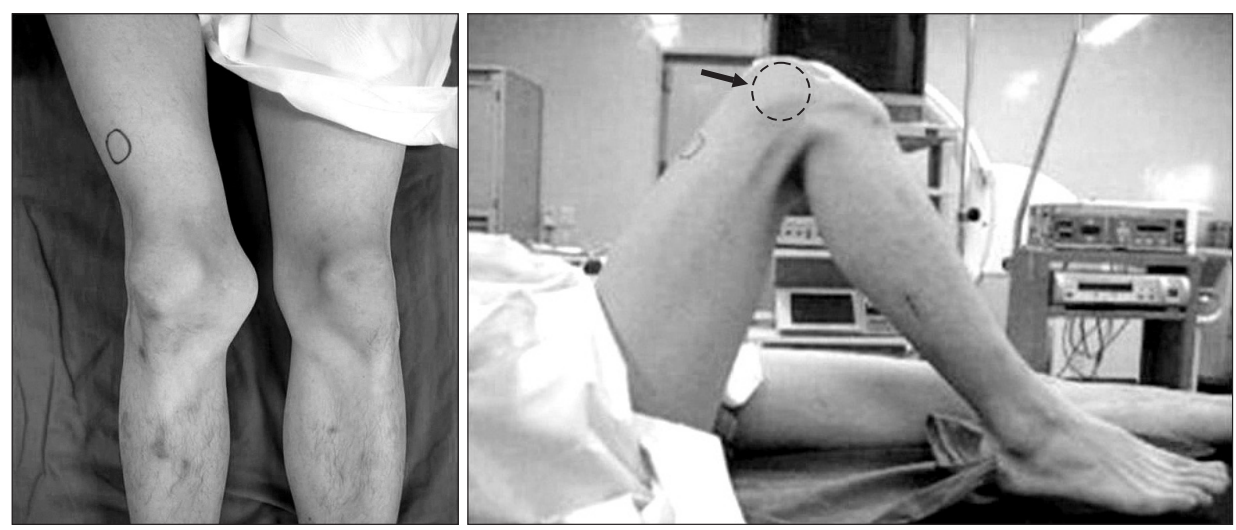

Fig. 1. Patella is dislocated when the knee is flexed and reduced in extension.
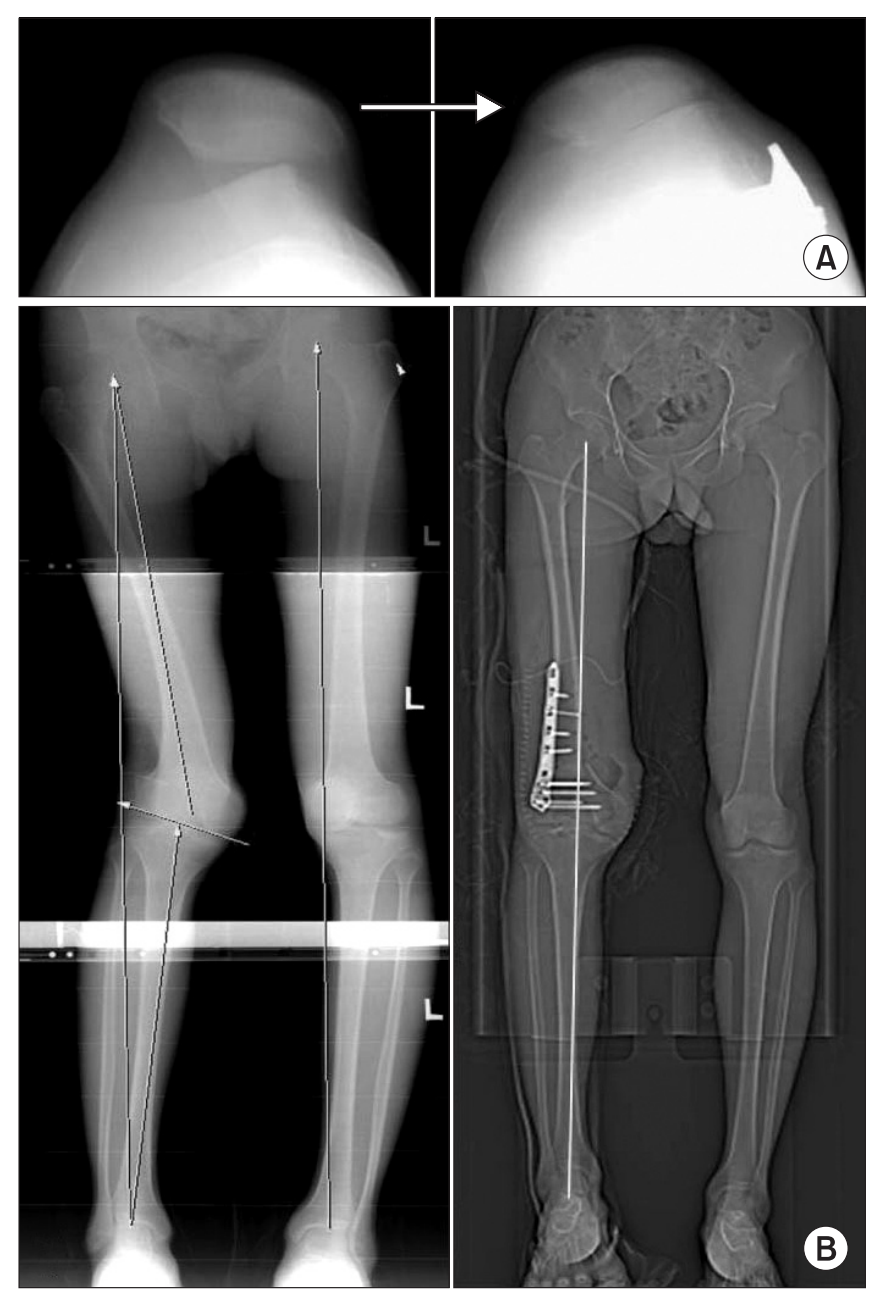

Fig. 2. (A) Two year after surgery, normal patella-femoral alignment was achieved. (B) Distal femoral varus osteotomy was performed.

$3^{\text {rd }}$ postoperative week. Weight bearing was gradually permitted and complete weight bearing was allowed at the $3^{\text {rd }}$ postoperative month.

On the postoperative radiograph, the congruence angle, sulcus angle, Q-angle, and Insall-salvati ratio was $15.75^{\circ}, 152^{\circ}, 2.3^{\circ}$, and 1.03 , respectively. The tibiofemoral angle was corrected to $4^{\circ}$ of valgus on the weight-bearing radiograph. Dislocation did not recur at 27 months after surgery. No limitation of joint motion was noted. The apprehension test for recurrent dislocation was negative (Fig. 2). The Kujala score ${ }^{1)}$ was 96 , indicating excellent clinical results.

\section{Discussion}

The causes of habitual patellar dislocation include contracture and fibrosis of the quadriceps femoris, vastus lateralis, and lateral retinaculum, abnormal iliotibial band attachment, repeated intramuscular injections into the thigh, patella alta due to the abnormal position of an elongated patellar tendon, systemic ligament laxity, dysplastic lateral femoral condyle, and genu valgum $^{2-4)}$.

A variety of surgical techniques have been introduced for the treatment of habitual dislocation of the patella. However, it has been known that combined procedures, not one single procedure, should be performed to achieve relatively satisfying treatment results.

Hnevkovsky et al. ${ }^{4)}$ attributed habitual patellar dislocation to contracture of the quadriceps femoris and introduced a technique to lengthen the muscle with the use of a lateral retinacular release and quadricepsplasty, which did not bring about complete flexion in their patients. In our patient, genu valgum and lateral femoral condyle hypoplasia following a growth plate fracture were thought as the cause of the dislocation. A quadricepsplasty was considered unnecessary because he had no contracture of the quadriceps femoris. However, lateral retinacular release alone was not sufficient to achieve joint reduction.

Realignment procedures can be performed additionally to reduce the joint. They are broadly classified into proximal and 
distal realignment procedures or bone and soft tissue realignment procedures. Proximal soft tissue realignment procedures including lateral release and medial reefing and distal realignment procedures are commonly used in adults. In particular, distal realignment procedures are limited to adult patients with $\geq 20^{\circ}$ of Q-angle because they could result in premature growth plate closure in children with open growth plates.

Proximal realignment is the most effective treatment for reduction of patellar dislocation. However, vastus medialis advancement can cause an increase in pressure on the knee, which eventually results in patellofemoral arthritis. Recently, medial patellofemoral ligament reconstruction using semitendinosus or gracillis tendon is recommended ${ }^{5}$.

Although the patient's medial retinaculum was extensively elongated, it was thick enough to be reefed and sutured. Therefore, a reconstruction using a medial patellofemoral ligament allograft was not considered necessary and the reduction was maintained at the follow-up examination.

Appropriate surgical treatment methods for habitual dislocation of the patella with genu valgum still remain controversial. Gao et al. ${ }^{6)}$ reported that flexion contracture of the quadriceps muscle was the cause of lateral dislocation of the patella in children and the sustained contracture also resulted in genu valgum. A release of quadriceps femoris contracture can be effective in correcting genu valgum in children who have hypoplasia of the lateral femoral condyle due to growth plate damage ${ }^{7)}$. Still, the ideal age for the treatment has yet to be established.

In adults, if genu valgum deformity remains after realignment due to the unstable patella, symptoms may also persist. Coventry ${ }^{8)}$ reported that femoral supracondylar varus osteotomy should be performed in patients with $\geq 12^{\circ}$ of symptomatic genu valgum or with $\geq 10^{\circ}$ of tilt of the articular surface. Healy et al. ${ }^{9)}$ recommended to perform corrective osteotomy of the distal femur for $\geq 15^{\circ}$ of genu valgum. Shen et al..$^{10)}$ suggested proximal soft tissue realignment and distal femoral osteotomy and anteromedial tibial tubercle transfer to treat genum valgum with $\geq 20^{\circ}$ of femorotibial angle. The femorotibial angle was $16^{\circ}$ in our patient. Therefore, we thought proximal soft tissue realignment alone was not sufficient to realign the patella and performed a femoral supracondylar osteotomy in addition. Femoral supracondylar osteotomy for genu valgum can be performed using an open or closed technique, but the latter has been associated with postoperative joint stiffness ${ }^{7}$. Considering that the risk of joint stiffness would be high in our patient because of the complex procedures including lateral retinacular release and medial reefing, we used an open osteotomy technique and took care not to damage the joint capsule during surgery.

We believe that clinical improvement of habitual dislocation of the patella can be obtained with correction of soft tissue imbalance and contracture and realignment of the patellofemoral and tibiofemoral joints. In particular, soft tissue procedures should be followed by bony procedures including femoral supracondylar osteotomy in cases of habitual dislocation of the patella with genu valgum.

\section{References}

1. Kujala UM, Jaakkola LH, Koskinen SK, Taimela S, Hurme M, Nelimarkka O. Scoring of patellofemoral disorders. Arthroscopy. 1993;9:159-63.

2. Gunn DR. Contracture of the Quadriceps Muscle. A Discussion on the Etiology and Relationship to Recurrent Dislocation of the Patella. J Bone Joint Surg Br. 1964; 46:4927.

3. Williams PF. Quadriceps contracture. J Bone Joint Surg Br. 1968;50:278-84.

4. Hnevkovsky O, Prague, Czechoslovakia. Progressive fibrosis of the vastus intermedius muscle in children: a cause of limited knee flexion and elevation of the patella. J Bone Joint Surg Br. 1961;43:318-25.

5. Fulkerson JP. Diagnosis and treatment of patients with patellofemoral pain. Am J Sports Med. 2002;30:447-56.

6. Gao GX, Lee EH, Bose K. Surgical management of congenital and habitual dislocation of the patella. J Pediatr Orthop. 1990;10:255-60.

7. Deie M, Ochi M, Sumen Y, Yasumoto M, Kobayashi K, Kimura H. Reconstruction of the medial patellofemoral ligament for the treatment of habitual or recurrent dislocation of the patella in children. J Bone Joint Surg Br. 2003;85:887-90.

8. Coventry MB. Upper tibial osteotomy for osteoarthritis. J Bone Joint Surg Am. 1985;67:1136-40.

9. Healy WL, Anglen JO, Wasilewski SA, Krackow KA. Distal femoral varus osteotomy. J Bone Joint Surg Am. 1988; 70:102-9.

10. Shen HC, Chao KH, Huang GS, Pan RY, Lee CH. Combined proximal and distal realignment procedures to treat the habitual dislocation of the patella in adults. Am J Sports Med. 2007;35:2101-8. 